\title{
Bioinformatics analysis of single and multi-hybrid epitopes of GRA-1, GRA-4, GRA-6 and GRA-7 proteins to improve DNA vaccine design against Toxoplasma gondii
}

\author{
Minoo Shaddel $^{1} \cdot$ Mansour Ebrahimi $^{1,2}$ (1) $\cdot$ Mohammad Reza Tabandeh $^{3}$
}

Received: 20 March 2018/Accepted: 10 April 2018/Published online: 17 April 2018

(C) Indian Society for Parasitology 2018

\begin{abstract}
Toxoplasma gondii, is a causative agent of morbidity and mortality in immunocompromised and congenitally-infected individuals. Attempts to construct DNA vaccines against $T$. gondii using surface proteins are increasing. The dense granule antigens are highly expressed in the acute and chronic phases of $T$. gondii infection and considered as suitable DNA vaccine candidates to control toxoplasmosis. In the present study, bioinformatics tools and online software were used to predict, analyze and compare the structural, physical and chemical characters and immunogenicity of the GRA-1, GRA-4, GRA- 6 and GRA-7 proteins. Sequence alignment results indicated that the GRA-1, GRA-4, GRA-6 and GRA-7 proteins had low similarity. The secondary structure prediction demonstrated that among the four proteins, GRA-1 and GRA-6 had similar secondary structure except for a little discrepancy. Hydrophilicity/hydrophobicity analysis showed multiple hydrophilic regions and some classical high hydrophilic domains for each protein sequence. Immunogenic epitope prediction results demonstrated that the GRA-1 and GRA-4 epitopes were stable and GRA-4 showed the highest degree of antigenicity. Although the GRA-7 epitope had the highest score of immunogenicity, this epitope was instable and had the lowest degree of antigenicity and half-time in eukaryotic cell. Also, the
\end{abstract}

Mansour Ebrahimi

Mansour.ebrahimi91@yahoo.com

1 Department of Parasitology, Faculty of Medicine, Aja University of Medical Sciences, Tehran, Iran

2 Department of Pathobiology, Faculty of Veterinary Medicine, Shahid Chamran University of Ahvaz, Ahvaz, Iran

3 Department of Basic Sciences, Faculty of Veterinary Medicine, Shahid Chamran University of Ahvaz, Ahvaz, Iran results indicated that GRA4-GRA7 epitope and GRA6GRA7 had the highest degree of antigenicity and immunogenicity among multi-hybrid epitopes, respectively. Totally, in the present study, single epitopes showed the highest degree of antigenicity compared with multihybrid epitopes. Given the results, it can be concluded that GRA-4 and GRA-7 can be powerful DNA vaccine candidates against $T$. gondii.

Keywords Bioinformatics analysis - Toxoplasma gondii . GRA proteins - DNA vaccine

\section{Introduction}

The intracellular protozoan, Toxoplasma gondii, is an opportunistic parasite infecting all warm-blooded animals including human beings (Shaddel et al. 2014b). Although T. gondii often causes subclinical infection, it may cause neuropsychological manifestations including hydrocephalus, blindness, mental retardation and encephalitis and high rates of morbidity and mortality in congenitallyinfected and immunocompromised patients (Shaddel et al. 2007; Sanecka et al. 2016). Furthermore, T. gondii infection may lead to remarkable economic losses due to stillbirths, abortion, and neonatal deaths in livestock which is a main source of the infection to human (Bai et al. 2012; Shaddel et al. 2014a). Therefore, the development of effective vaccines against this pathogen would be a crucial purpose to control the infection and reduce economic losses in the livestock industry (Meng et al. 2012). DNA vaccine could be an ideal technique against $T$. gondii because it is safer, able to induce a wider range of immune response types and easy to produce. DNA-based vaccination is considered as one of the most efficient strategies 
against intracellular parasites (Dumonteil 2007). Efforts to identify and construct DNA vaccine against $T$. gondii using surface proteins as ideal targets are increasing (Zhou et al. 2016). Based on the results from many studies, the most promising vaccine candidates of $T$. gondii include the dense granule (GRA), surface antigens (SAG proteins), rhoptry and microneme proteins (Couper et al. 2003; Martin et al. 2004).

It has been reported that the GRA antigens play a vital role in parasite survival and virulence and in forming the parasitophorous vacuole (Mercier et al. 2005; Mozaffarian et al. 2014). These proteins are highly expressed in the acute and chronic phases of the infection and can stimulate host immunity responses. Several GRA antigens in particular GRA-1, GRA-4, GRA6 and GRA-7 have been considered as suitable DNA vaccine candidates to control toxoplasmosis (Bivas-Benita et al. 2003) but how to decide which of these proteins should be chosen for in vivo studies remains a challenge. The identification of protein epitopes characters using bioinformatics tools will be useful for diagnostic purposes and development of DNA vaccine (Wang et al. 2013).

Bioinformatics, as a nexus of computer science, mathematics and biotechnology, is one of the newest scientific fields involved in biological issues. Bioinformatics approaches present useful results from large amounts of raw data (Romano et al. 2011). They have been extensively used to analyze gene and protein expression and predict structural, immunogenicity and general features of proteins. Study and comparison of the physical, chemical and immunogenicity characteristics of proteins can increase our knowledge regarding them and help the researchers select the proper epitopes for DNA vaccine investigations.

Therefore, the present study aimed at analyzing and comparing the structural, physical and chemical characters and immunogenicity of the GRA-1, GRA-4, GRA- 6 and GRA-7 proteins using bioinformatics approaches.

\section{Materials and methods}

\section{Data resources}

The protein sequences of $T$. gondii GRA-1, GRA-4, GRA-6 and GRA-7 were obtained from ToxoDB 10.0 (http://tox odb.org/toxo/). T-Coffee server (http://tcoffee.crg.cat/apps/ tcoffee/do:regular) was used to align the protein sequences. The secondary Structure of proteins was predicted using SOPMA secondary structure prediction method, available at (https://npsa-prabi.ibcp.fr/cgi-bin/npsa_automat.pl?page =npsa_sopma.html).

\section{Determination of immunogenic epitope of each protein}

In order to determine the immunogenic epitopes of GRA-1, GRA-4, GRA-6 and GRA-7 proteins, different parameters including hydrophilicity (Parker et al. 1986), beta turn prediction (Chou and Fasman 1979), antigenicity (Kolaskar and Tongaonkar 1990), flexibility (Karplus and Schulz 1985) and surface accessibility (Emini et al. 1985) were separately calculated for each protein by Immune Epitope Database (IEDB) Analysis resources (http://tools.iedb.org/bcell/). The epitopes with high score were then selected. The general features of the proteins including molecular weight, the estimated half-life, amino acid composition and stability index were predicted by Prot-Param (http://web.expasy.org/ protparam/). The selected epitopes of proteins were then linked together using a linker to form different multi-hybrid epitopes.

\section{Comparison of the single and multi-hybrid epitopes}

To determine the proper epitopes for DNA vaccine study against $T$. gondii, all single and multi-hybrid epitopes of the GRA proteins were compared together based on their immunogenicity, secondary structure and general features using mentioned software and bioinformatics tools.

\section{Results}

\section{Protein information and Sequence Alignment Analysis of the GRA proteins}

The GRA-1, GRA-4, GRA- 6 and GRA-7 genes encoded proteins of 190, 345, 179 and 236 amino acids, respectively. The molecular weight (MW) and Theoretical isoelectric point $(\mathrm{T} \mathrm{pI})$ of the proteins were as follow: GRA-1 (MW: $20 \mathrm{kDa}, \mathrm{T}$ pI: 4.15), GRA-4 (MW: $36 \mathrm{kDa}, \mathrm{T}$ pI: 7.05), GRA-6 (MW: $18 \mathrm{kDa}, \mathrm{T}$ pI: 6.75) and GRA-7 (MW: $26 \mathrm{kDa}, \mathrm{T}$ pI: 5.08).

Multiple sequence alignment of GRA-1, GRA-4, GRA-6 and GRA-7 showed that the four proteins sequences had low similarity (Fig. 1).

\section{Secondary Structure for GRA-1, GRA-4, GRA-6 and GRA-7 Proteins}

SOPMA method was used to predict the secondary structures of the proteins. Figure 2 showed that GRA-1 protein has 12 alpha helices, 6 extended strands, 9 -turn and 14 coils; GRA-4 protein has 10 alpha helices, 13 extended strands, 15 -turn and 19 coils; GRA-6 protein has 11 alpha helices, 7 extended strands, 9 -turn and 15 coils, and GRA- 


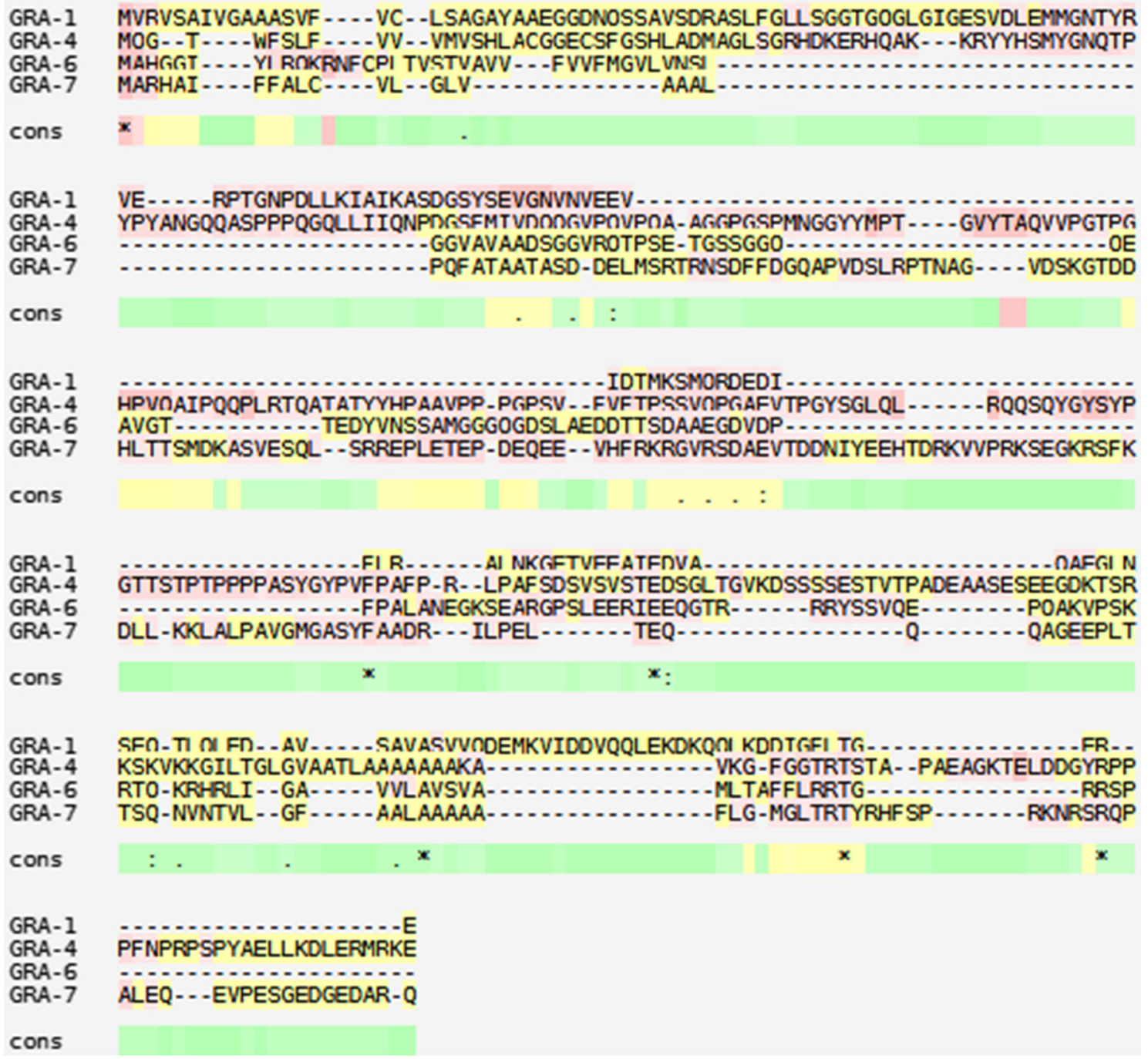

Fig. 1 Protein sequence alignment of GRA-1, GRA-4, GRA-6 and GRA-7 using T-Coffee

7 protein has 10 alpha helices, 6 extended strands, 5 -turn and 13 coils.

Results indicated that GRA- 1 and GRA- 6 have similar secondary structure except for a little discrepancy.

\section{Prediction of hydrophilicity}

In the present study, the hydrophilic/hydrophobic regions of the proteins were predicted using the scale Hphob./ Abraham \& Leo in the ProtScale program. Multiple hydrophilic regions and some classical high hydrophilic domains of each epitope can be observed in Fig. 3.

\section{Prediction of immunogenic epitopes}

The immunogenicity of the GRA-1, GRA-4, GRA-6 and GRA-7 epitopes was predicted by IEDB Analysis resources (Fig. 4). This program evaluates immunogenicity of the epitopes based on parameters described previously and extracts the data as score. Numeral indexes of the halftime, stability index, antigenicity property and immunogenicity of the single (Table 1) and multi-hybrid epitopes (Table 2) of GRA-1, GRA-4, GRA-6 and GRA-7 proteins are summarized.

As observed in Table 1, the GRA-1 and GRA-4 epitopes are stable and GRA-4 shows the highest degree of antigenicity. Although the GRA-7 epitope has the highest score of immunogenicity, this epitope is instable and has the lowest degree in antigenicity and half-time in eukaryotic cell. Based on data presented in Table 2, the only stable multi-hybrid epitope is GRA1-GRA4. Also, the results show that GRA4-GRA7 epitope and GRA6-GRA7 have the highest degree of antigenicity and immunogenicity, respectively. Given the information presented in this study, GRA-4 epitopes seems to be the most 
Hhhhhhhhhhhhheeeeecttchhhttccccchhhhhhhhheeeettccccccccccheehhhhttcee

GRA-1 eccccccctheeeeehcttcccceeccclhhhhhhhhhhhctthhhhhhhhcttchhhhhhhhhhhhtt

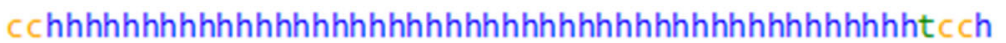

Etchhhhheeeeehhhctttccccchhhhhhhttttcchhhhhhhhhhhhhheecccccccccccccc cccccceeeeecttcceeeettcccccccccccccccceecccleeeeecttccccceeccc

GRA-4 cccccccreeescccccccccceeescccccttccccttccteeeeccccccccccccccccccccc ccccccccccttccccceeeeeccttceeecccccccccchhhhhhhhhhtccccccccctte

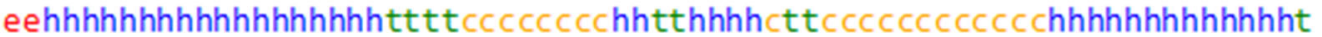

Hctteeeeccttccccee hheeeeeehheehhtttceeeccttccccccccccccccchheccc

GRA-6 hheehhhccttccccccccccchhhhttcccccchhhhttcccccccchhhhhhhttccccccccccc

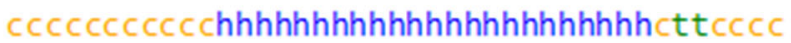

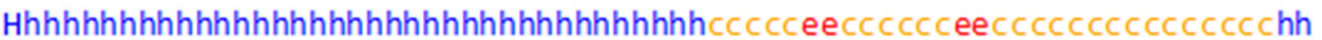

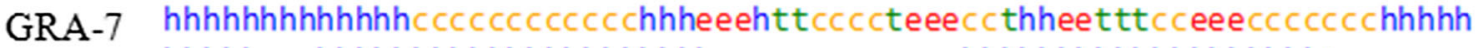

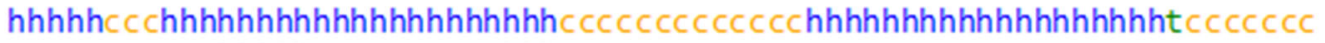
ccccccccchhhhheccecttcchhh

Fig. 2 Secondary structure analysis of the GRA proteins according to the SOPMA secondary structure prediction method. h: alpha helix, c: random coil regions, e: extended strand regions, t: -turn

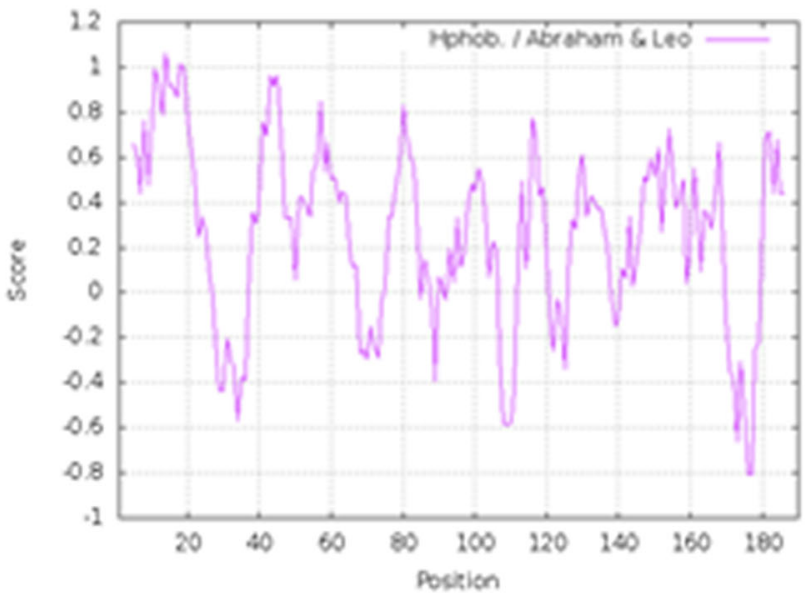

GRA-1

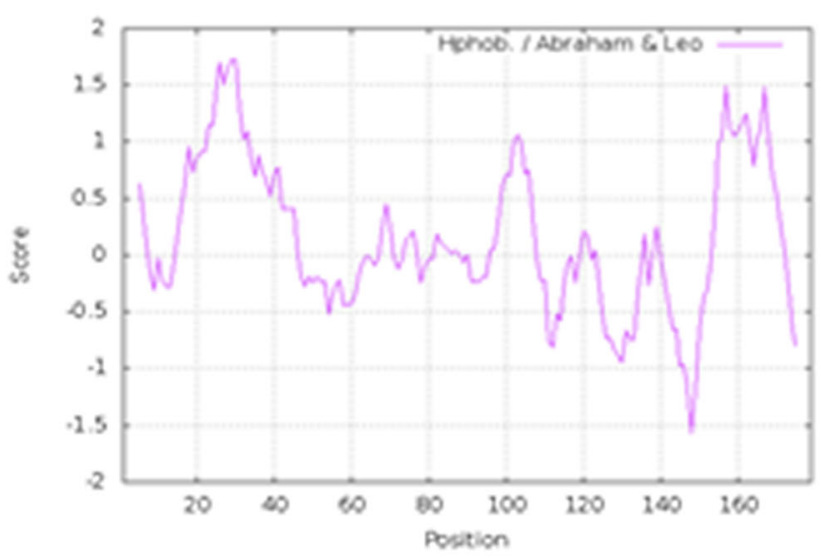

GRA-6

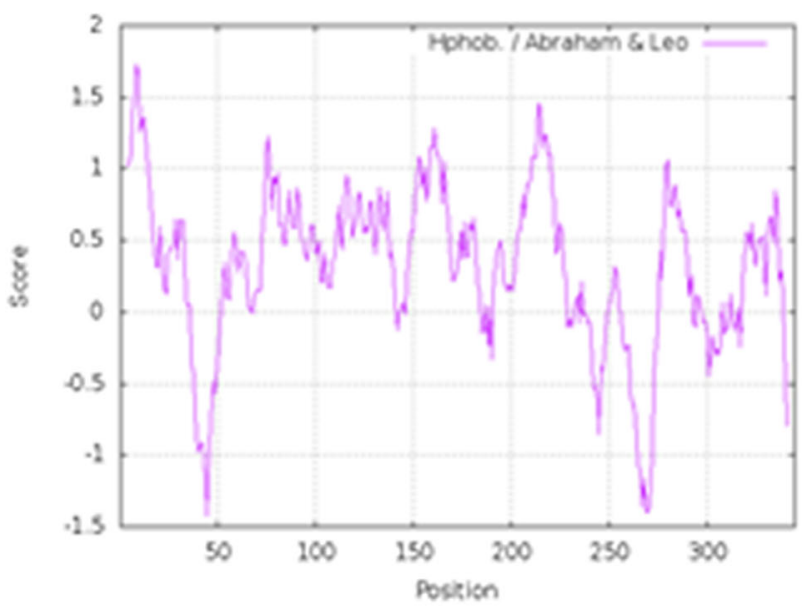

GRA-4

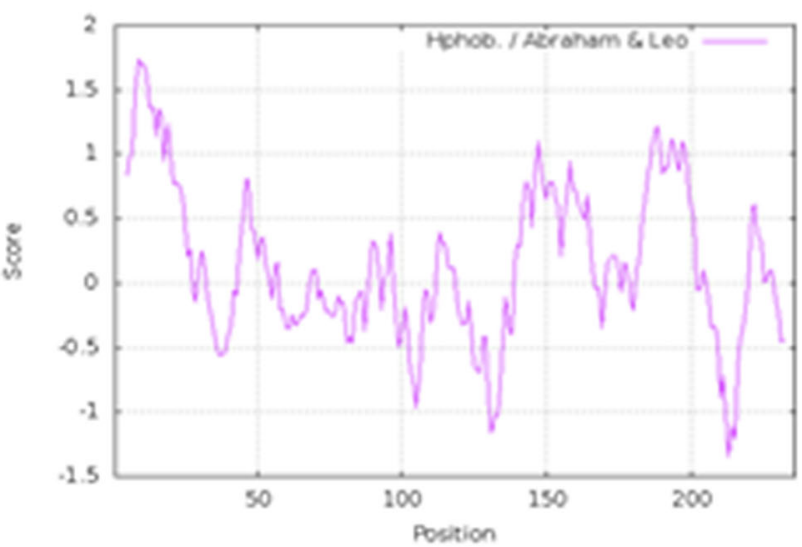

GRA-7

Fig. 3 The hydrophilicity/hydrophobicity of the GRA proteins 
Fig. 4 Immunogenic epitopes of the GRA-1, GRA-4, GRA-6 and GRA-7 proteins

\begin{tabular}{|c|c|c|}
\hline \hline Position & Sequence & Score \\
\hline $23-32$ & YAAEGGDNOS & 1.77 \\
\hline $43-50$ & GLLSGGTG & 1.22 \\
\hline $68-74$ & YRVERPT & 1.57 \\
\hline $83-90$ & IAIKASDGS & 1.44 \\
\hline $104-110$ & DTMIKSMO & 1.54 \\
\hline $169-175$ & OOLEKDK & 1.80 \\
\hline $183-189$ & GFLTGER & 1.20 \\
\hline
\end{tabular}

GRA-1

\begin{tabular}{|c|c|c|}
\hline Position & Sequence & Score \\
\hline $33-43$ & AGLSGRHDKER & $\mathbf{1 . 8 2}$ \\
\hline $79-84$ & LIIONPD & $\mathbf{0 . 9 6 7}$ \\
\hline $100-106$ & OAAGGPG & 1.48 \\
\hline $193-200$ & YSYPGTTS & $\mathbf{1 . 4 9}$ \\
\hline $228-234$ & VSVSTED & $\mathbf{1 . 7 5}$ \\
\hline $236-248$ & GLTGVKDSSSSES & $\mathbf{2 . 2 1}$ \\
\hline $254-273$ & $\begin{array}{c}\text { DEAASESEEGDK } \\
\text { TSRKSKVK }\end{array}$ & $\mathbf{2 . 3 1}$ \\
\hline
\end{tabular}

GRA-4

\begin{tabular}{|c|c|c|c|c|c|}
\hline Position & Sequence & Score & Position & Sequence & Score \\
\hline $5-12$ & GI & & $32-40$ & DELMSRTRN & 1.77 \\
\hline \multirow{2}{*}{$42-62$} & \multirow{2}{*}{$\begin{array}{l}\text { VAADSGGVRQTPS } \\
\text { ETGSSGGO }\end{array}$} & \multirow[b]{2}{*}{1.93} & $56-65$ & TNAGVDSKGT & 1.62 \\
\hline & & & $76-84$ & ASVESOLSR & 1.76 \\
\hline $76-83$ & SAMGGGOG & 1.56 & $98-106$ & VHFRKRGVR & .77 \\
\hline $85-92$ & EDDTT & 1.42 & \multirow{2}{*}{$116-136$} & \multirow{2}{*}{\begin{tabular}{|c|} 
IYEEHTDRKVVPR \\
KSEGKRSF
\end{tabular}} & \multirow{2}{*}{1.72} \\
\hline $104-114$ & PALANEGKSEA & 1.70 & & & \\
\hline $121-132$ & ERIEEQGTRRRY & 1.82 & $205-217$ & YRHFSPRKNRSRQ & 1.91 \\
\hline $138-150$ & POAKVPSKRTOKR & 1.81 & $222-231$ & QEVPESGEDG & 1.94 \\
\hline
\end{tabular}

Table 1 Immunogenicity, antigenicity and general features of the single epitope of GRA-1, GRA-4, GRA-6 and GRA-7 proteins (using ProtParam, Predicted Antigenic Peptides software)

\begin{tabular}{lllllll}
\hline Protein & Instability & Half-time (eukaryote) & Half-time (prokaryote) & Half-time (fungi) & Antigenicity & Immunogenicity \\
\hline GRA-1 & Stable (24.01) & $2.8 \mathrm{~h}$ & $2 \mathrm{~min}$ & $10 \mathrm{~min}$ & 1.021 & 1.5 \\
GRA-4 & Stable (23.48) & $4.4 \mathrm{~h}$ & $10 \mathrm{~h}$ & $20 \mathrm{~h}$ & 1.029 & 1.66 \\
GRA-6 & Instable (66.3) & $30 \mathrm{~h}$ & $10 \mathrm{~h}$ & $20 \mathrm{~h}$ & 1.022 & 1.65 \\
GRA-7 & Instable (54.49) & $1.1 \mathrm{~h}$ & $10 \mathrm{~h}$ & $3 \mathrm{~min}$ & 1.010 & 1.78 \\
\hline
\end{tabular}

suitable candidate for DNA vaccine study among the studied epitopes.

\section{Discussion}

Bioinformatics approaches play an undeniable role in vaccine development to predict protein structures, functions, and other biological characteristics and to understand the specific immune response of selected vaccine epitopes (Lundegaard et al. 2010; Zhang et al. 2013). One of the main goals of the bioinformatics is to increase our knowledge about the biological processes and improve the health level using new vaccines design. DNA vaccination is a powerful technique with strong potential to induce specific humoral and cellular immune responses (Hiszczynska-Sawicka et al. 2011). Given the importance of toxoplasmosis in human health and also recent developments in designing DNA vaccine based on GRA proteins against this disease, we carried out a study to analyze and compare the structural, physical and immunogenicity features of the single and multi-epitopes of GRA-1, GRA-4, GRA-6 and GRA-7 proteins using bioinformatics tools and websites to design proper DNA vaccine against $T$. gondii. To the best of our knowledge, there have been no reported studies about the bioinformatics analysis of GRA proteins 
Table 2 Immunogenicity, antigenicity and general features of the multi-hybrid epitope of GRA-1, GRA-4, GRA-6 and GRA-7 proteins (using ProtParam, Predicted Antigenic Peptides software)

\begin{tabular}{llccccc}
\hline Epitope & Instability & Half-time (eukaryote) $(\mathrm{h})$ & Half-time (prokaryote) (h) & Half-time (fungi) & Antigenicity & Immunogenicity \\
\hline GRA4-GRA1 & Stable & 4.4 & 10 & $20 \mathrm{~h}$ & 0.975 & 1.58 \\
GRA4-GRA7 & Instable & 4.4 & 10 & $20 \mathrm{~h}$ & 0.981 & 1.72 \\
GRA6-GRA1 & Instable & 100 & 10 & $20 \mathrm{~h}$ & 0.974 & 1.57 \\
GRA6-GRA4 & Instable & 100 & 10 & $20 \mathrm{~h}$ & 0.976 & 1.65 \\
GRA6-GRA7 & Instable & 100 & 10 & $20 \mathrm{~h}$ & 0.979 & 1.71 \\
GRA7-GRA1 & Instable & 1.1 & 10 & $3 \mathrm{~min}$ & 0.980 & 1.64 \\
GRA6-GRA4-GRA1 & Instable & 100 & 10 & $20 \mathrm{~h}$ & 0.974 & 1.60 \\
GRA4-GRA7-GRA1 & Instable & 4.4 & 10 & $20 \mathrm{~h}$ & 0.978 & 1.65 \\
GRA6-GRA4-GRA7 & Instable & 100 & 10 & $20 \mathrm{~h}$ & 0.978 & 1.70 \\
\hline
\end{tabular}

in Iran and there are few researches regarding the subject in other countries. Bioinformatics analyses of protein structure to predict the immunity contributed to the development of a new vaccine candidate against $T$. gondii (Cong et al., 2010).

In the present study, hydrophilicity/hydrophobicity of the proteins was predicted. Analysis of amino acid hydrophilicity/hydrophobicity presents suitable information to understand the protein folding, analyze the protein interaction sites and predict the protein secondary structure (Bai et al. 2012). The secondary structures have a major impact on the epitopes. The $\alpha$-helices and $\beta$-turn, located in the inner parts of the protein, have a high hydrogen-bond energy which can preserve the structure of a protein and make strong interactions with antibodies. We use IEDB to predict the immunogenicity of the epitopes based on five parameters including hydrophilicity, beta turn prediction, antigenicity, flexibility and surface accessibility. Wang et al. (2014) reported that these methods reliably predicted the proper epitopes for DNA vaccine development. The IEDB Analysis Resource database uses NetMHC pan as prediction method since 2011. This method generates a quantitative prediction of the affinity of any peptide-MHC class I interaction, covering HLA-A and HLA-B for humans as well as chimpanzee, macaque, gorilla, cow, pig and mouse. (Hoof et al. 2009). Hydrophilicity, beta-turns, secondary structure, and surface accessibility are the important features of the amino acids which can present significant and useable data of epitopes for biological studies including DNA vaccine. (Wee et al. 2010; Zhao and Li 2010). Epitope prediction methods are mostly based on several features (not one) of the proteins because researchers believe that only by evaluation one character we cannot access to sufficient and accurate information regarding the epitope (Kringelum et al. 2012).

In the present study, among single and multi-epitopes, GRA-4 showed the highest degree of antigenicity. Given the scores obtained from the studied parameters, GRA-4 seems to be a proper antigen to use as a target in developing of DNA vaccine against $T$. gondii. This protein is considered as one of the most important $T$. gondii's surface antigens that has been used to design DNA vaccine. The main epitope in GRA-4 structure is epitope B which includes 11 amino acids from the C terminal of GRA-4. The second most important epitope of the GRA-4 is located between amino acids 318 and 334 (Mévélec et al. 1998). In the present study, for GRA-4 epitopes, amino acids 236-273 showed the highest degree of immunogenicity. Meisel et al. (1996) reported that in GRA-4 protein, amino acids ranging from 229-242 to 231-245 induce cellular and humoral immunity against $T$. gondii. The GRA4 231-245 peptide is immunogenic and considered a suitable target for DNA vaccine design (Wang et al. 2014). We demonstrated that peptide 205-231 amino acids of GRA-7 had the greatest degree of immunogenicity based on studied parameters. However, stability index and the estimated half-time of this epitope were lower than other studied proteins.

In the current study, single epitopes had higher degree of antigenicity compared with multi-epitopes. Nevertheless, some studies reported that multi-epitope antigens are of the most important antigens for the development of diagnostic kits for toxoplasmosis screening (Dai et al. 2012). Among the studied multi-epitopes, GRA7-GRA1 epitope showed high degree of immunogenicity. This finding is consistent with the results recorded by Jongert et al. (2007). Moreover, immunization of $T$. gondii-infected mice with DNA vaccine encoding GRA-1 and GRA-7 leads to protective immune responses and reduction of brain cysts. DNA vaccination with GRA1-GRA7 (in mixture form) induced protection against infection with different virulent $T$. gondii strains in mice (Jongert et al. 2007).

Bioinformatics analysis in the present study indicated that the GRA-6, one of the favorite antigens for DNA 
vaccine researches against $T$. gondii, had the longest halftime compared with other epitopes. GRA-6 is a highly immunogenic candidate to design vaccine against toxoplasmosis. This protein is the source of the immunodominant HF10 peptide which is located at the carboxyl terminus of GRA6 and presented by the H-2Ld major histocompatibility complex class I molecule (MHC class I). It induces a protective immune response in $\mathrm{BALB} / \mathrm{c}$ mice carrying the $\mathrm{H}-2 \mathrm{Ld}$ molecule against $T$. gondii infection (Nicolas and Federico 2008).

The GRA6 gene encodes a 230-amino-acid polypeptide and does not contain any introns. The deduced polypeptide contains two hydrophobic regions with the characteristics of transmembrane domains. The central hydrophobic domain is surrounded by two hydrophilic domains which contain four blocks of amino acids. The C-terminal hydrophilic region comprises $24 \%$ of glycine residues indicating a structural role for GRA6 in the network (Lecordier et al. 1995).

Bioinformatics can play a significant role in study of antigenic proteins, by which we can understand the structures and functions of an antigen and select proper epitopes without running the risks involved in cultivating the pathogen of interest. Epitopes prediction can reveal the pathogenesis and immune mechanisms of pathogens, and prepare crucial data for the development of diagnostic reagents and design of immunogenic peptides and new vaccines (Bai et al. 2012). This approach presents some privileges such as faster output and less cost compared with traditional vaccinology methods (Tong and Ren 2009).

\section{Conclusion}

The present study showed the information of structure and basic characteristics of the GRA proteins. The results from the current study should contribute to the development of a highly protective DNA vaccine against $T$. gondii. Taking account of the predicted results, it can be concluded that GRA-4 and GRA-7 are potential proteins with strong antigenicity; hence, they can be powerful DNA vaccine candidates against $T$. gondii. The present study provided a theoretical basis for the screening of effective DNA vaccine candidate components against infection with $T$. gondii. The results of the present study should be considered in further experimental researches to determine the suitable GRA epitopes for DNA vaccine study against $T$. gondii.

Acknowledgement We would like to acknowledge the Aja University of Medical Sciences management for supporting us to do this study.
Author contributions All authors contributed extensively to the present study. M.S, M.E and MR.T designed the study; M.E and MR.T collected and analyzed Data; M.E and M.S wrote the main manuscript. All authors discussed the results and commented on the manuscript at all stages.

Funding This study was funded by Aja University of Medical Sciences (Grant No. 695520).

\section{Compliance with ethical standards}

Conflict of interest The authors declare that there is no conflict of interest.

Ethical approval This article does not contain any studies with human participants or animals performed by any of the authors.

\section{References}

Bai Y, He S, Zhao G, Chen L, Shi N, Zhou H, Cong H, Zhao Q, Zhu XQ (2012) Toxoplasma gondii: bioinformatics analysis, cloning and expression of a novel protein TgIMP1. Exp Parasitol 132:458-464. https://doi.org/10.1016/j.exppara

Bivas-Benita M, Laloup M, Versteyhe S, Dewit J, De Braekeleer J, Jongert E, Borchard G (2003) Generation of Toxoplasma gondii GRA1 protein and vaccine loaded chitosan particles: preparation, characterization and preliminary in vivo studies. Int $\mathrm{J}$ Pharm 266(1-2): 17-27

Chou PY, Fasman GD (1979) Prediction of beta-turns. Biophys J 26:367-383

Cong H, Mui EJ, Witola WH, Sidney J, Alexander J, Sette A, Maewal A, McLeod R (2010) Human immunome, bioinformatic analyses using HLA supermotifs and the parasite genome, binding assays, studies of human $\mathrm{T}$ cell responses, and immunization of HLAA*1101 transgenic mice including novel adjuvants provide a foundation for HLA-A03 restricted CD8 + T cell epitope based, adjuvanted vaccine protective against Toxoplasma gondii. Immunome Res 6:12

Couper KN, Nielsen HV, Petersen E, Roberts F, Roberts CW, Alexander J (2003) DNA vaccination with the immunodominant tachyzoite surface antigen (SAG-1) protects against adult acquired Toxoplasma gondii infection but does not prevent maternofoetal transmission. Vaccine 21:2813-2820

Dai JF, Jiang M, Wang YY, Qu LL, Gong RJ, Si J (2012) Evaluation of a recombinant multiepitope peptide for serodiagnosis of Toxoplasma gondii infection. Clin Vaccine Immunol 19:338-342

Dumonteil E (2007) DNA vaccines against protozoan parasites: advances and challenges. $\mathrm{J}$ Biomed Biotechnol. https://doi.org/10.1155/2007/90520

Emini EA, Hughes JV, Perlow DS, Boger J (1985) Induction of hepatitis A virus-neutralizing antibody by a virus-specific synthetic peptide. J Virol 55:836-839

Hiszczyńska-Sawicka E, Olędzka G, Holec-Gąsior L, Li H, Xu JB, Sedcole R, Kur J, Bickerstaffe R, Stankiewicz M (2011) Evaluation of immune responses in sheep induced by DNA immunization with genes encoding GRA1, GRA4, GRA6 and GRA7 antigens of Toxoplasma gondii. Vet Parasitol 177:281-289

Hoof I, Peters B, Sidney J, Pedersen LE, Sette A, Lund O, Buus S, Nielsen M (2009) NetMHCpan, a method for MHC class I binding prediction beyond humans. Immunogenetics 61:1-13 
Jongert E, de Craeye S, Dewit J, Huygen K (2007) GRA7 provides protective immunity in cocktail DNA vaccines against Toxoplasma gondii. Parasite Immunol 29:445-453

Karplus PA, Schulz GE (1985) Prediction of chain flexibility in proteins. Naturwissenschaften 72:212-213

Kolaskar AS, Tongaonkar PC (1990) A semi-empirical method for prediction of antigenic determinants on protein antigens. FEBS Letters 276:172-174

Kringelum JV, Lundegaard C, Lund O, Nielsen M (2012) Reliable B cell epitope predictions: impacts of method development and improved benchmarking. PLoS Comput Biol 8:12

Lecordier L, Moleon-Borodowsky I, Dubremetz JF, Tourvieille B, Mercier C, Deslée D, Capron A, Cesbron-Delauw MF (1995) Characterization of a dense granule antigen of Toxoplasma gondii (GRA6) associated to the network of the parasitophorous vacuole. Mol Biochem Parasitol 70(1-2):85-94

Lundegaard C, Hoof I, Lund O, Nielsen M (2010) State of the art and challenges in sequence based T-cell epitope prediction. Immunome Res 6(Suppl 2):S3. https://doi.org/10.1186/1745-75806-s2-s3

Martin V, Supanitsky A, Echeverria PC, Litwin S, Tanos T, De Roodt AR, Guarnera EA, Angel SO (2004) Recombinant GRA4 or ROP2 protein combined with alum or the gra4 gene provides partial protection in chronic murine models of toxoplasmosis. Clin Diagn Lab Immunol 11:704-710

Meisel R, Stachelhaus S, Mevelec MN, Reichmann G, Dubremetz JF, Fischer HG (1996) Identification of two alleles in the GRA4 locus of Toxoplasma gondii determining a differential epitope which allows discrimination of type versus type II and III strains. Mol Biochem Parasitol 81:259-263

Meng M, He S, Zhao G, Bai Y, Zhou H, Cong H, Lu G, Zhao Q, Zhu XQ (2012) Evaluation of protective immune responses induced by DNA vaccines encoding Toxoplasma gondii surface antigen 1 (SAG1) and 14-3-3 protein in BALB/c mice. Parasit Vectors 5:273

Mercier C, Adjogble DKZ, Däubener W, Cesbron-Delauw MF (2005) Dense granules: are they key organelles to help understand the parasitophorous vacuole of all apicomplexa parasites? Int $\mathrm{J}$ Parasitol 35:829-849

Mévélec MN, Mercereau-Puijalon O, Buzoni-Gatel D, Bourguin I, Chardès T, Dubremetz JF, Bout D (1998) Mapping of B epitopes in GRA4, a dense granule antigen of Toxoplasma gondii and protection studies using recombinant proteins administered by the oral route. Parasite Immunol 20:183-195

Mozaffarian F, Khoshdel AR, Shaddel M (2014) Threat of toxoplasmosis targeting the brain for military forces and veterans. Arch Mil Med 3:e18560
Nicolas B, Federico G (2008) Immunodominant, protective response to the parasite Toxoplasma gondii requires antigen processing in the endoplasmic reticulum. Nat Immunol 9:937-944

Parker JM, Guo D, Hodges RS (1986) New hydrophilicity scale derived from high-performance liquid chromatography peptide retention data: correlation of predicted surface residues with antigenicity and $\mathrm{x}$-ray-derived accessible sites. Biochemistry 25:5425-5432

Romano P, Giugno R, Pulvirenti A (2011) Tools and collaborative environments for bioinformatics research. Brief Bioinform 12:549-561. https://doi.org/10.1093/bib/bbr055

Sanecka A, Nagisa Y, Dougan SK, Jackson J, Shastri N, Ploegh H, Blanchard N, Frickel EM (2016) Transnuclear CD8 T cells specific for the immunodominant epitope Gra6 lower acutephase Toxoplasma gondii burden. Immunology 149:270-279

Shaddel M, Mehbod ASA, Karamy M (2007) Toxoplasma gondii infection in neonates. Iran J Parasitol 3:34-37

Shaddel M, Mirzaii-Dizgah I, Hoshangi M (2014a) Anti-Toxoplasma gondii antibody levels in blood supply of Shiraz Blood Transfusion Institute, Iran. Iran J Parasitol 9:120-124

Shaddel M, Mirzaii Dizgah I, Sharif F (2014b) The prevalence of toxoplasmosis in Imam Reza Hospital blood bank samples, Tehran, Iran. Transfus Apher Sci 51:181-183

Tong JC, Ren EC (2009) Immunoinformatics: current trends and future directions. Drug Discov Today 14:684-689

Wang YH, Wang GX, Zhang DL, Yin H, Wang M (2013) Identification of novel B cell epitopes within Toxoplasma gondii GRA1. Exp Parasitol 135:606-610

Wang Y, Wang G, Ou J, Yin H, Zhang D (2014) Analyzing and identifying novel B cell epitopes within Toxoplasma gondii GRA4. Parasit Vectors 7:474

Wee LJK, Simarmata D, Kam YW, Ng LFP, Tong JC (2010) SVMbased prediction of linear B-cell epitopes using Bayes Feature Extraction. BMC Genom 4:S21. https://doi.org/10.1186/14712164-11-S4-S21

Zhang NZ, Chen J, Wang M, Petersen E, Zhu XQ (2013) Vaccines against Toxoplasma gondii: new developments and perspectives. Expert Rev Vaccines 12:1287-1299

Zhao L, Li J (2010) Mining for the antibody-antigen interacting associations that predict the B cell epitopes. BMC Struct Biol 1:S6. https://doi.org/10.1186/1472-6807-10-S1-S6

Zhou J, Wang L, Zhou A, Lu G, Li Q, Wang Z, Zhu M, Zhou H, Cong $\mathrm{H}$, He S (2016) Bioinformatics analysis and expression of a novel protein ROP48 in Toxoplasma gondii. Acta Parasitol 61:319-328 